\title{
Temporal stability of posterior EEG alpha over twelve years
}

\author{
Craig E. Tenke ${ }^{a, b, c}$, Jürgen Kayser ${ }^{a, b, c, *}$, Jorge E. Alvarenga ${ }^{a}$, Karen S. Abraham ${ }^{a}$, \\ Virginia Warner ${ }^{\mathrm{b}, \mathrm{d}}$, Ardesheer Talati ${ }^{\mathrm{b}, \mathrm{c}, \mathrm{d}}$, Myrna M. Weissman ${ }^{\mathrm{b}, \mathrm{c}, \mathrm{d}}$, Gerard E. Bruder $^{\mathrm{a}, \mathrm{c}}$ \\ ${ }^{a}$ Division of Cognitive Neuroscience, New York State Psychiatric Institute, New York, NY, USA \\ ${ }^{\mathrm{b}}$ Division of Translational Epidemiology, New York State Psychiatric Institute, New York, NY, USA \\ ${ }^{c}$ Department of Psychiatry, Columbia University College of Physicians E Surgeons, New York, NY, USA \\ ${ }^{\mathrm{d}}$ Mailman School of Public Health, Columbia University, New York, NY, USA
}

\section{A R T I C L E I N F O}

\section{Article history:}

Accepted 20 March 2018

Available online 16 April 2018

\section{Keywords:}

EEG

Temporal stability

Trait biomarker

Surface Laplacian

Current source density (CSD)

Principal components analysis (PCA)

\section{H I G H L I G H T S}

- Evaluates test-retest reliability of posterior EEG alpha at rest in recordings separated by 12 years.

- Reliability was excellent in adults for overall alpha and acceptable for net and overall asymmetry.

- Reliability was substantially weaker in children or adolescents (aged <18 at first test session).

\section{A B S T R A C T}

Objective: We previously identified posterior EEG alpha as a potential biomarker for antidepressant treatment response. To meet the definition of a trait biomarker or endophenotype, it should be independent of the course of depression. Accordingly, this report evaluated the temporal stability of posterior EEG alpha at rest.

Methods: Resting EEG was recorded from 70 participants (29 male; 46 adults), during testing sessions separated by $12 \pm 1.1$ years. EEG alpha was identified, separated and quantified using reference-free methods that combine current source density (CSD) with principal components analysis (PCA). Measures of overall (eyes closed-plus-open) and net (eyes closed-minus-open) posterior alpha amplitude and asymmetry were compared across testing sessions.

Results: Overall alpha was stable for the full sample (Spearman-Brown $\left[r_{\mathrm{SB}}\right]=.834$, Pearson's $r=.718$ ), and showed excellent reliability for adults $\left(r_{\mathrm{SB}}=.918 ; r=0.848\right)$. Net alpha showed acceptable reliability for adults $\left(r_{\mathrm{SB}}=.750 ; r=.600\right)$. Hemispheric asymmetries (right-minus-left hemisphere) of posterior overall alpha showed significant correlations, but revealed acceptable reliability only for adults $\left(r_{\mathrm{SB}}=.728 ; r=.573\right)$. Findings were highly comparable between 29 male and 41 female participants. Conclusions: Overall posterior EEG alpha amplitude is reliable over long time intervals in adults. Significance: The temporal stability of posterior EEG alpha oscillations at rest over long time intervals is indicative of an individual trait.

(C) 2018 International Federation of Clinical Neurophysiology. Published by Elsevier B.V. All rights reserved.

\section{Introduction}

The hope offered by biomarkers and endophenotypes (Beauchaine, 2009) of antidepressant treatment response rests on the elimination or reduction of costly treatment delays by supplementing or replacing trial-and-error testing with individualized treatment (Trivedi et al., 2016). Resting EEG activity in the alpha

\footnotetext{
* Corresponding author at: New York State Psychiatric Institute, Unit 50, 1051 Riverside Drive, New York, NY 10032, USA.

E-mail address: kayserj@nyspi.columbia.edu (J. Kayser).
}

and theta bands is predictive of a positive response to a range of antidepressants (for reviews, see Alhaj et al., 2011; Bruder et al., 2013). Greater alpha power, particularly at posterior scalp sites, has been reported in patients who respond to antidepressants compared to those who do not (Tenke et al., 2011; Ulrich et al., 1986), especially over right hemisphere sites (Bruder et al., 2008). Owing to these clinical interests, the temporal stability of EEG measures has been frequently studied on a time scale conforming to typical treatment protocols, with high test-retest reliability of EEG alpha and theta power reported for depressed patients (Bruder et al., 2008: 12 weeks) as well as healthy adults 
(Enoch et al., 2008: 2 years; Smit et al., 2005: 1.8 years; Tomarken et al., 1992: 3 weeks; Tenke et al., 2017a: 1 week).

In marked contrast to the brain changes that underlie clinical depression or treatment outcome, an endophenotype should precede the onset of the disorder and persist independent of its course or the outcome following treatment (Beauchaine, 2009; Gottesman and Gould, 2003; Gould and Gottesman, 2006). Consequently, individual differences in EEG measures should be demonstrable and persistent not just in clinical populations, but in preclinical and healthy populations as well, with trait-like stability over substantial periods of time. High test-retest correlations have been reported for broad band spectral amplitude measures at time scales of months (Salinsky et al., 1991), and for spectral patterns over 1-3 years (Näpflin et al., 2007).

This report assessed the temporal stability of posterior EEG alpha in a sample of participants in a longitudinal study of familial risk of major depressive disorder (MDD), with EEG testing sessions separated by a span of twelve years. Following our previous recommendations (Tenke et al., 2017a), posterior EEG alpha was identified and quantified using reference-free measures that combine current source density (CSD) frequency spectra with principal components analysis (CSD-fPCA; Tenke and Kayser, 2005; Tenke et al., 2011, 2013, 2017a, 2017b). Given prior evidence that overall alpha amplitude is in part unrelated to the classical Berger (1929) effect, that is, the condition-dependent blockade of resting alpha with eyes open as opposed to closed (net alpha; Tenke et al., 2015), we evaluated both aspects of posterior alpha as core characteristics of the resting EEG paradigm.

\section{Methods}

\subsection{Participants}

Participants ( $N=70,41$ female, 59\%) were part of a multiwave, three-generation longitudinal study of individuals at high and low risk for major depression based on family history (Weissman et al., $1997,2005,2016 a, 2016 b)$. In the original wave of the study, probands with moderate to severe major depressive disorder were selected from outpatient clinics for the psychopharmacologic treatment of mood disorders, and nondepressed, demographicallymatched control participants were selected from an epidemiologic sample of adults with no psychiatric history from the same community. The sample was recruited from an urban setting (greater New Haven area, Connecticut, US), and consisted of Caucasian, working or middle class individuals. Clinical assessments were conducted by independent interviewers who were blind to the participant's previous clinical history (personal or family). All assessments were approved by the institutional review boards at Yale University and at Columbia University/New York State Psychiatric Institute (NYSPI). Written informed consent was obtained from all participants, or from the legal guardian for minors.

Participants were included in this study solely based on the availability of EEG data at both time points, in marked contrast to cross-sectional analyses from this sample aimed at distinguishing EEG differences reflecting familial risk status for MDD, generation, lifetime history of MDD, and interview items associated with protection against, or resilience following, MDD (Bruder et al., 2005, 2007; Tenke et al., 2013, 2017b). Collection of resting EEG was added to the study protocol at Wave 4 (approximately 20year time point at Yale) and repeated at Wave 6 (30-year time point at Columbia/NYSPI). The mean time interval elapsed between test and retest was $12 \pm 1.1$ years (range 9.4-15.5 years). Most participants were children $(n=34)$ or grandchildren $(n=29)$ of the proband, with approximately equal representation of high $(n=33)$ and low $(n=30)$ familial risk status. Participants at high familial risk had significantly greater lifetime rates of MDD at Wave $6(60.6 \%)$ than those in the low risk group (30.5\%; Fisher exact test, one-sided, $p=.027$ ). The remaining seven participants were unrelated to either proband (i.e., they married-into the study); however, as family risk status is of secondary importance for the primary objective of evaluating the temporal stability of posterior EEG alpha, these individuals were included in the overall analysis to increase sample size and statistical power. Wave 4 EEG from 18 of these participants (Tenke et al., 2013; $N=52$ ), and Wave 6 EEG from 46 participants (Tenke et al., 2017b; $N=73$ ) were included in previous reports using comparable EEG methods.

The participants' mean ages were $26 \pm 13$.3 years (range $=5.2-$ 47.6) at Wave 4 and $38 \pm 13.2$ years (range $=17.1-59.2$ ) at Wave 6. To account for anticipated developmental differences in EEG, temporal stability was computed separately for the full current sample $(N=70)$ and for an adult subsample ( $\geq 18$ years; $n=46$ ).

\subsection{EEG methods}

As many aspects of EEG acquisition, processing and analysis have been detailed in prior reports (e.g., Tenke et al., 2011, 2013, 2017a, 2017b), we will present a brief overview with methodological details that are unique to this report.

\subsubsection{Resting EEG paradigm}

EEG at rest was measured while participants sat quietly during four 2-min periods (order of eyes-open and eyes-closed counterbalanced across participants and assessments) after being instructed to avoid blinking and eye or body movements (fixation cross used for eyes-open condition; e.g., Tenke et al., 2011).

\subsubsection{Wave 4: EEG acquisition}

Thirteen scalp EEG channels based on the 10/20 system (4 frontal: F7/8, F3/4; 5 central: T7/8, C3/4, Cz; 4 parietal: P7/8, P3/4), two EEG reference channels (right and left ear, digitally re-referenced to linked ears), and bipolar recordings to monitor blinks (above vs. below right eye) and horizontal eye movements (right vs. left canthi) were recorded at Yale University using an electrode cap (Electro Cap International; see Tenke et al., 2013). The EEG was acquired using a Bioamplifier system (James Long Company) at a gain of $10 \mathrm{~K}$ and a band pass of .01-30 Hz. EEG data were continuously acquired at 200 samples/s (NeuroScan, 2003) and segmented off-line into consecutive 1.28 -s epochs every .64 s (50\% overlap). Epochs contaminated by blinks, eye movements, or movementrelated artifacts were excluded using a rejection criterion of $\pm 100 \mu \mathrm{V}$ on any channel, followed by interactive rejection of remaining artifacts (Bruder et al., 2005).

\subsubsection{Wave 6: EEG acquisition}

Continuous data (256 samples/s) were recorded at NYSPI using an Active2 recording system (BioSemi, 2001) with a 72-channel 10/10 system scalp montage (Jurcak et al., 2007; Pivik et al., 1993; see Tenke et al., 2017a) including the nose as a reference. Data were blink-corrected offline using a spatial, singular value decomposition (NeuroScan, 2003), and segmented into 2-s epochs (75\% overlap). Epoched data were screened for electrolyte bridges (Alschuler et al., 2014; Tenke and Kayser, 2001), and affected channels interpolated via spherical splines (Perrin et al., 1989). Additional EEG data were preserved by identifying isolated EEG channels containing artifacts or noise on any given epoch (e.g. amplifier drift, residual eye activity, muscle or movement-related artifacts) using a reference-free approach (Kayser and Tenke, 2006b; Tenke et al., 2017a), which were then replaced by spherical spline interpolations (Perrin et al., 1989) from artifact-free channels (i.e., artifacts identified in less than $25 \%$ of all channels for that epoch). Bipolar vertical and horizontal EOGs were interpolated via 
spherical splines using all EEG channels (see Kayser and Tenke, 2015a). Epochs exceeding $\pm 100 \mu \mathrm{V}$ in any channel (including uncorrected EOG) were rejected to conform to Wave 4 methods, and the montage was then reduced to the 13 channels common to both waves. For these reasons, despite notable differences data acquisition between Waves 4 and 6, both data sets have the same final common denominator (sparse 13-channel EEG montage, $\pm 100 \mu \mathrm{V}$ blink rejection threshold).

\subsection{CSD spectra}

Epoched data were transformed to CSD using a spherical spline surface Laplacian (spline flexibility constant $\mathrm{m}=4$, regularization constant $\lambda=10^{-5}, 50$ iterations; Perrin et al., 1989; Kayser and Tenke, 2006a, 2015b; for a Matlab-based implementation, see Kayser, 2009). The DC offset of each epoch was removed, and the EEG was tapered over the entire duration of each epoch (Wave 4: $1.28 \mathrm{~s}$; Wave 6: $2 \mathrm{~s}$ ) using a Hanning window (Bendat and Piersol, 1971). Wave 4 spectral resolution was $0.78 \mathrm{~Hz}$ (Tenke et al., 2013), while Wave 6 resolution was $0.25 \mathrm{~Hz}$ after padding with 1-s of zeros at the beginning and end of each epoch (Tenke et al., 2017a, 2017b). ${ }^{1}$ Averaged power spectra were computed from all remaining epochs for eyes-open and -closed conditions. Total seconds $\left(\mathrm{S}_{\text {tot }}\right)$ of nonoverlapping data were computed as:

$\mathrm{S}_{\text {tot }}=\mathrm{T}_{\mathrm{e}} * \mathrm{~N}_{\mathrm{e}} / \mathrm{N}_{\mathrm{o}}$

where $\mathrm{T}_{\mathrm{e}}$ denotes epoch duration (Wave 4: $1.28 \mathrm{~s}$; Wave 6: $2 \mathrm{~s}$ ), $\mathrm{N}_{\mathrm{e}}$ is the total number of epochs/participant, and $\mathrm{N}_{\mathrm{o}}$ is the number of overlapping epochs (Wave 4: 2; Wave 6: 4). Only participants with $\mathrm{S}_{\text {tot }}>17$ for each condition (eyes open/closed) in each wave were included. EEG quantification relied on the following number of nonoverlapping epochs $(M \pm \mathrm{SD}$, eyes open vs. closed): for Wave 4 , $182.3 \mathrm{~s} \pm 45.8$ vs. $135.7 \pm 71.2$; for Wave $6,185.8 \pm 38.7$ vs. 196.2 \pm 35.2 .

\subsection{Quantification of posterior alpha generators}

Posterior alpha was identified and quantified by CSD-fPCA as previously described (Tenke and Kayser, 2005; Tenke et al., 2011, 2013, 2017a, 2017b). Power spectra for Waves 4 and 6 were imported into Matlab and interpolated to a common spectral resolution of $0.78 \mathrm{~Hz}$ over a $0-50 \mathrm{~Hz}$ frequency range. Spectral topographies for each sample point were scaled by the standard deviation of posterior (i.e., parietal) power across subjects and conditions as recommended for data obtained from multiple acquisition systems and recording montages (Tenke et al., 2017a). Power spectra were then converted to amplitude spectra (square root transformation), the frequency range truncated to $1.56-39.8 \mathrm{~Hz}$ (i.e., after eliminating the first frequency value), and submitted to unrestricted, covariance-based frequency PCA (fPCA) followed by Varimax rotation of the covariance loadings (Kayser and Tenke, 2003). Each of the first four factors accounted for $>5 \%$ spectral variance (total variance $=89.5 \%$, with peaks at 1.56 (delta), 25.00 (EMG beta), 10.15 (high-frequency alpha) and $9.37 \mathrm{~Hz}$ (low-frequency alpha). To further simplify the factor structure (i.e., reduce factor overlap), amplitude spectra were reconstructed from the two alpha factors and the overlapping delta factor (i.e., alpha prefiltered), the resulting data further truncated to $1.56-19.5 \mathrm{~Hz}$, and submitted to a final covariance-based fPCA with Varimax rotation (see Tenke et al., 2011; Tenke et al., 2017a, 2017b).

\footnotetext{
${ }^{1}$ After zero padding epochs from 2 to $4 \mathrm{~s}$, data for Wave 6 were rescaled (2x) to correct for the corresponding impact on calculating power estimates.
}

\subsection{Temporal stability of EEG alpha}

Alpha amplitude topographies were computed from low- and high-frequency alpha scores, and subsequently pooled across medial parietal sites $(\mathrm{P} 3 / 4)$ for each wave $(4,6)$ and condition (eyesopen, -closed). Overall and net alpha were quantified from the sum across and difference between (eyes-closed-minus-open) conditions, respectively (Tenke et al., 2017b). Overall and net asymmetries (right hemisphere minus left hemisphere) were also computed for posterior alpha, but anterior asymmetries were not, owing to (i) the absence of identifiable generators for these factors (Tenke and Kayser, 2005; Tenke et al., 2011) and (ii) their poor temporal stability over a one-week interval (see Supplementary Table S1 in Tenke et al., 2017a). Test-retest reliability (temporal stability) was evaluated from Pearson's $r$ and the corresponding Spearman-Brown ${ }^{2}$ for the full sample, as well as for 46 participants who were at least 18 years of age at the first EEG testing session (Wave 4). ${ }^{3}$

\section{Results}

Fig. 1 shows the common CSD-fPCA solution (factor loadings and mean factor score topographies) across Waves 4 and 6. Results were consistent with previous studies in yielding distinct spectral components over posterior regions separating conditiondependent (eyes-closed greater than eyes-open) high-frequency alpha ( $10.15 \mathrm{~Hz}$ peak frequency) from low-frequency alpha/theta $(9.37 \mathrm{~Hz}$ ). The remaining low-frequency activity (peak at $1.56 \mathrm{~Hz}$ ) was neither posterior nor condition-dependent. Posterior alpha topographies (sum across high and low alpha factors) were similar for Waves 4 and 6 (Fig. 2), with maxima at P3/4 for eyes-closed and overall (eyes-closed plus eyes-open) alpha.

Scatterplots of Waves 4 and 6 alpha measures and their corresponding regression lines were constructed to visualize temporal stability. Fig. 3 illustrates the stability of overall posterior alpha, which was further supported by a robust correlation (Pearson's $r$ $=.718, p<.001)$ and good reliability $\left(r_{\mathrm{SB}}=.836\right)$. However, 24 of these participants were children at Wave 4 (filled circles, solid line), 14 of which were below 13 yrs of age, thereby contributing developmental variance to the temporal stability estimates. When the analyses were restricted to the 46 participants who were 18 or older at the first test session (open circles, dashed line), the testretest correlation was considerably greater $(r=0.848)$, corresponding to excellent reliability $\left(r_{\mathrm{SB}}=.918\right)$. Moreover, further restriction to the 39 participants related to the original probands (Fig. 4) revealed a significantly reduced correlation for high vs. low risk ( $r=.740$ vs. .918, $z=1.694, p<.05,1$-tailed), despite their similar regression plots (solid vs. dashed lines).

Good or excellent reliabilities were also observed separately for the two conditions (eyes-open vs. eyes-closed, full sample: $r_{\mathrm{SB}}=$ .845 vs. .808 ; for adults only: $r_{\mathrm{SB}}=.907$ vs. .893 ). Correlations of net alpha (eyes closed-minus-open) were less robust for the full sample $\left(r=.531 ; r_{\mathrm{SB}}=.694\right.$, indicative of questionable reliability), although acceptable reliability was seen for the subsample of 46 adult participants $\left(r=.600 ; r_{\mathrm{SB}}=.750\right)$.

By contrast, hemispheric asymmetries of posterior alpha were substantially less stable over time. For the full sample, overall alpha asymmetry was significantly correlated between waves $(r$ $=.428)$ but had poor reliability $\left(r_{\mathrm{SB}}=.599\right)$, becoming acceptable only when restricted to the adult subsample $\left(r=.573, r_{\mathrm{SB}}=.728\right)$.

\footnotetext{
${ }^{2} r_{\mathrm{SB}}=(\mathrm{k} * r) /[1+(\mathrm{k}-1) * r]$, where $r$ denotes Pearson's $r, \mathrm{k}=2$.

3 The appropriateness of this age cutoff ( $\geq 18$ years) was supported by similar regression lines for adolescent participants ( $13-17$ years) and children ( $<13$ years).
} 

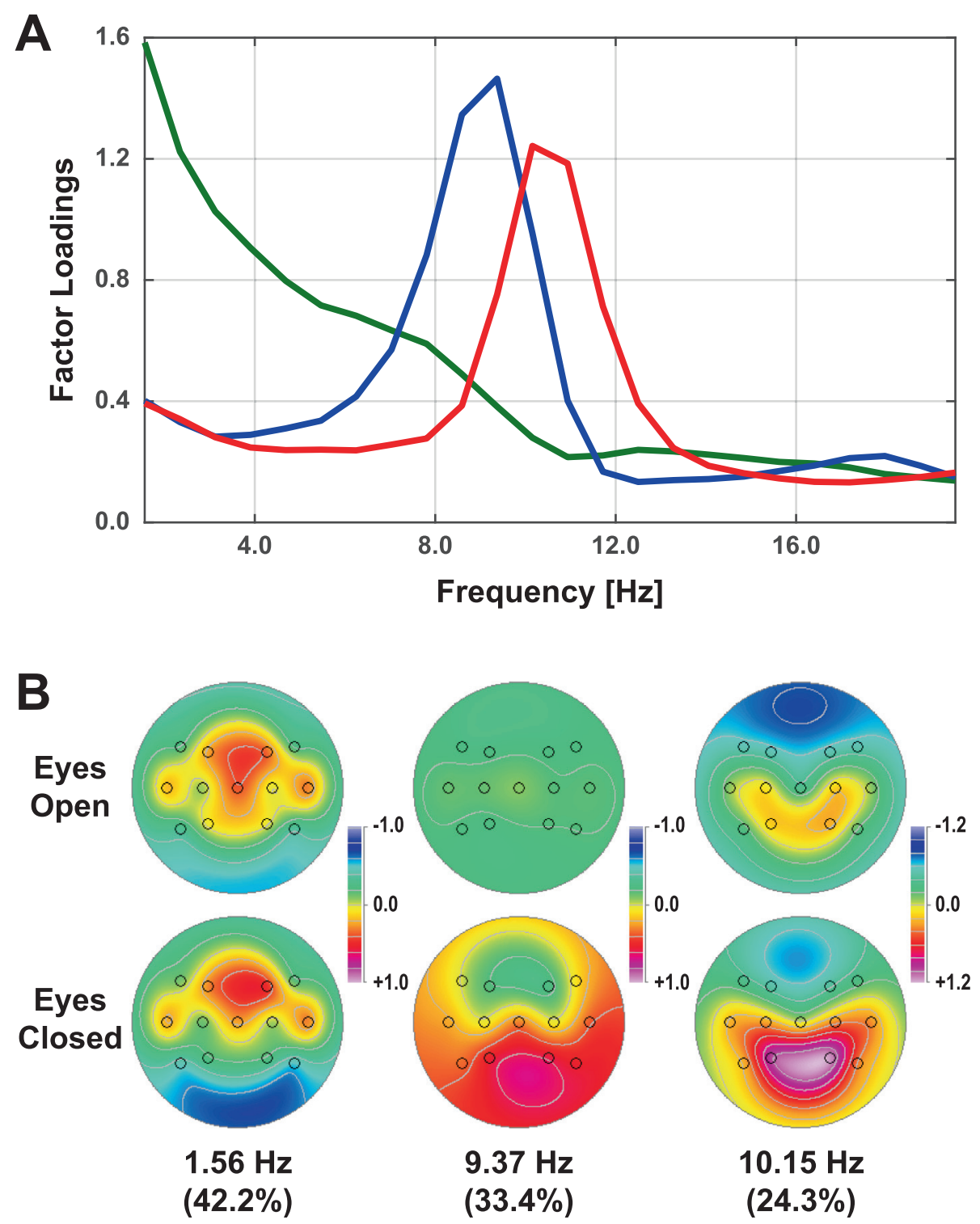

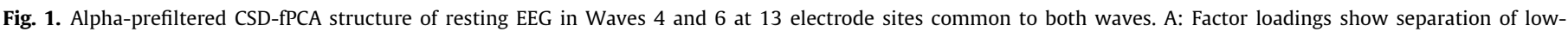

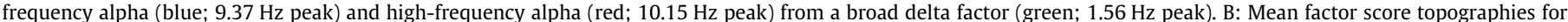

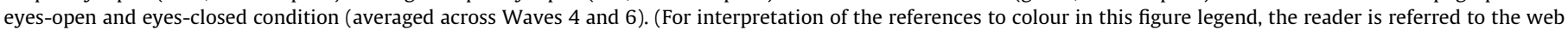
version of this article.)

The asymmetry of net alpha was unreliable both for the full sample $\left(r=.191, r_{\mathrm{SB}}=.321\right)$ and the adult subsample $\left(r=.322, r_{\mathrm{SB}}=.487\right)$.

Highly comparable reliabilities were seen across gender (29 male vs. 41 female, overall: $r_{\mathrm{SB}}=.768$ vs. .859 ; net: $r_{\mathrm{SB}}=.585$ vs. .782 ; eyes closed: $r_{\mathrm{SB}}=.735$ vs. .839 ; eyes open: $r_{\mathrm{SB}}=.780$ vs. .871 ), which revealed no significant differences between correlations (all $p>.20,2$-tailed). There were also no significant differences between correlations for adult participants (16 men vs. 30 women, all $p>.63,2$-tailed).

\section{Discussion}

\subsection{Overview}

Endophenotypes, as a subtype of biomarkers, should reflect persistent, trait-like properties (Beauchaine, 2009; Gould and Gottesman, 2006) that are not restricted to clinical populations or the timescale of treatment protocols. They should also appear throughout the population at large, and possibly throughout the life span after reaching brain maturity.

The present findings indicate strong temporal stability for the amplitude of posterior EEG alpha over a considerably greater time scale than previously reported. The unique longitudinal, multigenerational design of the data set demonstrates that overall posterior EEG alpha is a stable individual trait, showing at least acceptable temporal stability independent of risk status. However, comparisons of participants at low or high familial risk for depression suggest a slight decrease in stability with risk (i.e., $r^{2}$ indicates a decreased proportion of predicted variance from .84 to .54 ).

Although nominally somewhat higher in women, test-retest reliability was highly comparable for both genders, which further bolsters the interpretation as a trait biomarker.

These results have the additional advantages of CSD: they are not biased by volume conduction or choice of recording reference, but rather reflect the generator pattern at scalp underlying alpha oscillations. Moreover, the use of fPCA (Barry and De Blasio, 2017) and particularly CSD-fPCA provides a data-driven quantifica- 


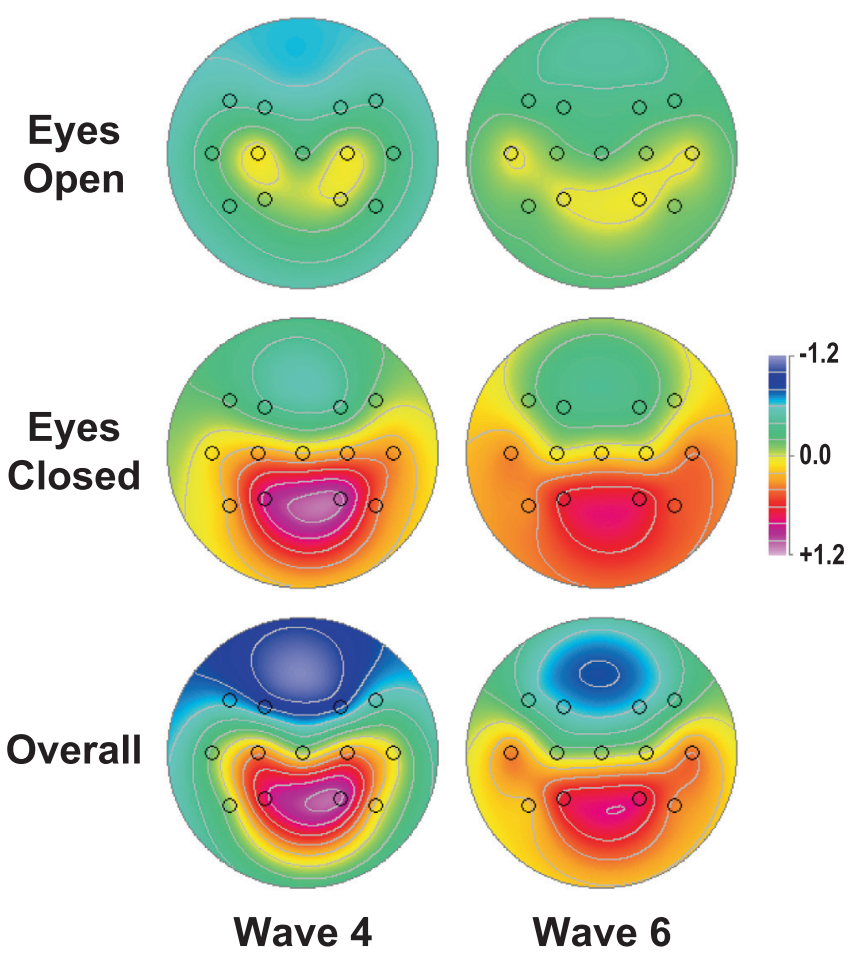

Fig. 2. Mean alpha topographies of factor scores (pooled across low- and highfrequency alpha) for eyes-open (top row), eyes-closed (middle row), and overall alpha (sum of eyes-open and eyes-closed; bottom row), separately shown for each wave.

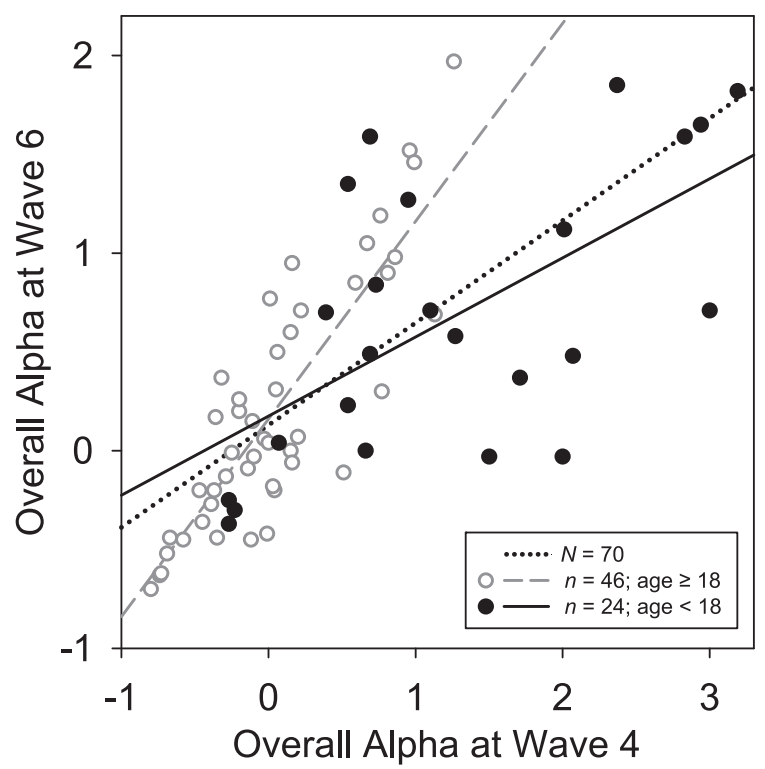

Fig. 3. Scatterplots of overall posterior alpha (factor scores pooled across low- and high frequency alpha, eyes open/closed conditions, and medial parietal sites) at Wave 4 and 6. Regression lines are shown for the full sample $(N=70, r=0.72$, dotted), as well as for adults (age $\geq 18$ years at Wave $4 ; n=46 ; r=0.85$, dashed, open circles) and children (age $<18$ years at Wave $4 ; n=24, r=0.61$, solid, filled circles).

tion of EEG alpha that is superior to rigidly defined spectral bands, while eliminating common sources of contamination and artifact (e.g., broadband EMG noise or residual EOG artifact; Tenke and Kayser, 2005; Tenke et al., 2011, 2017b). However, alpha or mu activity attributable to frontal or central regions is not represented by these measures of posterior alpha.

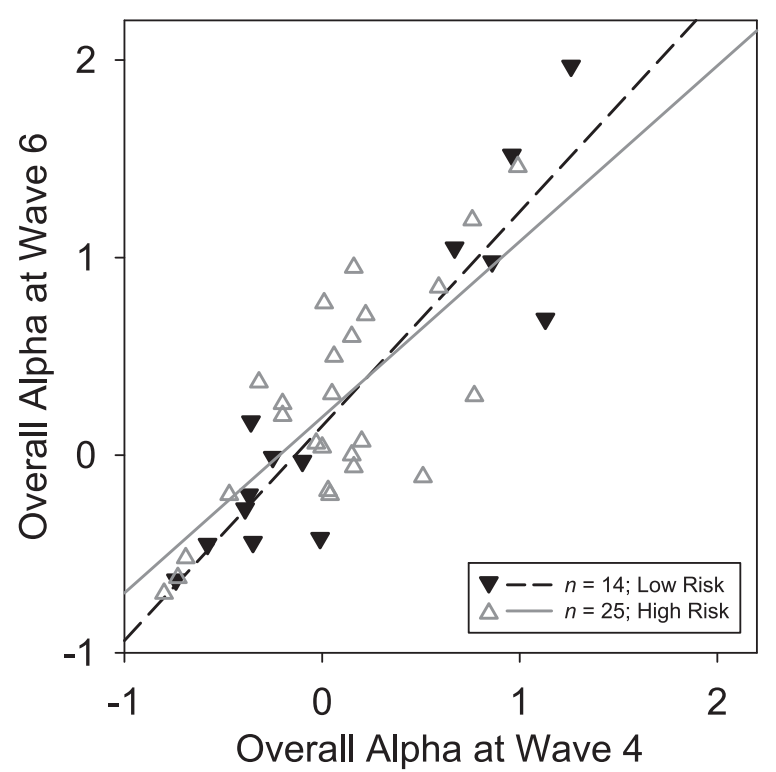

Fig. 4. Scatterplots as in Fig. 3 identifying adult participants (age $\geq 18$ years at Wave 4 ) by family risk status. Regression lines are shown for participants at high ( $n$ $=25, r=.74$, solid, open triangles $)$ or low risk $(n=14, r=.92$, dashed, filled triangles).

\subsection{Related findings in previous studies}

The standard resting EEG paradigm includes eyes-closed and eyes-open conditions, which allows the difference between conditions to reveal the classical Berger (1929) effect (i.e., alpha block or posterior net alpha). Viewed another way, reliance on net alpha provides a within-subjects baseline condition (i.e., eyes-open) for evaluating the spectrum and posterior topography of alpha. Although there are technical and statistical reasons to have confidence in alpha findings reported in this context (Bruder et al., 1997, 2005, 2007, 2008; Tenke et al., 2011), it is noteworthy that distributed generator solutions corresponding to midline frontal theta rely only on eyes-closed EEG (Korb et al., 2009; Pizzagalli et al., 2001; 2011). Since task-related alpha is more strongly correlated with overall than net alpha in healthy adults (Tenke et al., 2015), the notion that net alpha provides a superior estimate of individual alpha may not be generally supported.

At the same time, being a difference measure with additive errors resulting from separate alpha estimates for eyes-closed and -open conditions, net alpha is inherently noisier than overall alpha or either condition alone - a comparatively lower testretest reliability as observed here may therefore be expected. The same caveat applies to hemisphere asymmetry metrics, which are likewise inherently nosier than estimates pooled across both hemispheres. In case of net alpha asymmetries, the problem is compounded. The present reliability findings are consistent with these considerations.

We have previously suggested (Tenke et al., 2011) that prominent (i.e., high amplitude) posterior alpha might equally predict a positive outcome for a range of clinical antidepressant treatments, including selective serotonin reuptake inhibitor (SSRI) and possibly placebo responses, as well as spontaneous remissions. If true, posterior EEG alpha amplitude might be viewed as a trait biomarker associated with resilience vs. vulnerability. These considerations led us to compare posterior EEG alpha in individuals who differed in their self-reports of personal religion/spirituality (Tenke et al., 2013, 2017b), a measure which has been linked to protection against MDD (Miller et al., 1997, 2014). In line with these expectations, individuals with high importance ratings of 
religion/spirituality had greater overall posterior alpha than those who did not (Tenke et al., 2013). Moreover, the association between greater overall posterior alpha and high importance of religion/spirituality appeared to be linked to personal beliefs held early in life, suggesting that a critical developmental stage is linked to posterior resting alpha (Tenke et al., 2017b). These findings align with the present observations of temporal stability for overall posterior alpha being related to age at the first testing session, likely reflecting a link to brain development and maturation.

Apart from the hopes for EEG alpha in the development of individualized treatment for clinical depression, posterior, rightlateralized EEG alpha has been suggested as a potential marker for familial depression risk (Bruder et al., 2005, 2007). While the more nuanced topography expressed by an alpha asymmetry, generally with a specific focus on frontal asymmetries, has its own history in the literature on affective disorders, our methods provide no clear theoretical rationale for representing alpha at anterior sites. We consistently identify posterior, but not anterior, generators of alpha, and restrict our analyses to these generators. Despite this disclaimer, it is difficult to discount the spurious introduction of posterior alpha into the anterior field potential topography resulting from standard EEG reference schemes (Tenke and Kayser, 2015). It is also likely that the (phase-inverted) posterior topography of alpha may itself volume conduct to anterior sites (Tenke and Kayser, 2015), whereby the use of a log transformation will enhance it (i.e., log asymmetry measures are log ratios, regardless of amplitude). In both of these circumstances, field potential topographies that may be mistakenly attributed to a frontal origin will be correctly identified and quantified by our CSD-fPCA measures.

A final consideration is the previously reported association between low-frequency alpha/theta at posterolateral and anterior midline sites (see Fig. 5 of Tenke and Kayser, 2005). This activity undoubtedly contributes to the anterior topography of alpha, particularly as reflected in the poorly understood relationship between the identifying CSD-fPCA factor and anterior midline theta, as localized by distributed inverse solutions to rostral anterior cingulate cortex (Korb et al., 2009; Pizzagalli et al., 2001).

A separate indication of the association between the topography of posterior EEG alpha is its inverse relationship to cortical thinning (Bruder et al., 2012). Cortical thinning is a structural measure that has been suggested as an endophenotype for depression, and is likewise stable over time (Waves 5 and $6 ; 5-11$ years; Hao et al., 2017). Since high importance of religion/spirituality was linked to thicker posterior cortex (Miller et al., 2014), it is tempting to suggest that the structural and EEG measures reflect a common mechanism for risk and resilience for affective disorders. These possibilities cannot be further pursued at the present time, owing to the further reductions in sample size that arise from incomplete data for one or more modalities at any assessment time point during this longitudinal study.

\subsection{Limitations}

The observation that posterior alpha shows greatest temporal stability in adults is not surprising. In a longitudinal study of healthy children aged 4-17 spanning up to 7 years (mean 4.6 years), Benninger et al. (1984) reported a number of changes in the theta and alpha bands (average recording reference). In particular, occipital alpha increased with development, while theta decreased. The spectral EEG shift first appeared in low-frequency alpha, and later in high-frequency alpha, particularly in girls. Despite this shift, these authors noted that the "individual characteristics of the EEG spectra show[ed] a remarkable stability over the years." A preliminary inspection of the two alpha factors in the present study are consistent with these observations. While it could be argued that a complete description of the spectral variability requires an alternative quantitative strategy altogether, such as determining the individual alpha frequency (Doppelmayr et al., 1998), we note that in all but the youngest participants the developmental shift is adequately represented by the balance between low- and high-frequency alpha which jointly comprise posterior alpha. Since our previous findings do not support a separate contribution of the two alpha factors, either as indices of MDD treatment response (Tenke et al., 2011) or as the indirect influences represented by personal importance of Religion/Spirituality (Tenke et al., 2013), they are not discussed further.

One notable limitation is our use of CSD with a sparse electrode montage. The lack of occipital recording sites may be considered an additional concern for estimates of alpha. Although the adequacy of a low-density montage was previously evaluated (see Supplement 4 of Tenke et al., 2011), as well as the present montage in particular (see Supplement 1 of Tenke et al., 2013), we recognize that not all nuances of the CSD-fPCA factor structure identified for Wave 6 in Tenke et al. (2017b) are fully represented in the present montage. Despite this limitation, we have shown that common concerns over a loss of information with low-resolution CSD methods are exaggerated or misplaced when using standard computational methods (i.e., spherical spline interpolations; cf. Fig. 15 of Kayser and Tenke, 2015b; Tenke and Kayser, 2012). Most importantly, the two CSD-fPCA alpha factors extracted with this sparse montage closely reproduced the critical spectral (low- and highfrequency alpha), topographical (posterior maximum) and conditional (eyes-closed greater eyes-open) characteristics of analogous alpha factors extracted from a 72-channel EEG data set (e.g., see Fig. 1 in Tenke et al., 2011). To the extent that these lowresolution CSD measures are less robust than for the full 72channel montage of Wave 6 , the high reliability observed with the common Wave 4 montage is sufficient support for this approach until the next wave of high-resolution EEG has been acquired (i.e., Wave 7) and these concerns can be evaluated in an improved manner.

The extent to which differences in EEG recordings and data processing between session 1 and 2 (including blink artifact detection, reduction and/or removal) affected the reported results is unclear. However, if they did, one would expect a decrease in signal-tonoise ratio and therefore less temporal stability (i.e., an underestimation of test-retest reliability). Data processing relied on the optimal procedures available and appropriate for the respective EEG montage and system at that time to improve spectral estimates. The current focus on the high theta and alpha frequency range $(6-13 \mathrm{~Hz})$ is largely outside the spectral characteristics of blinks, as a typical blink duration lasts about $100-400 \mathrm{~ms}$ (i.e., a half cycle) or about 1-5 Hz. Moreover, the chosen analytical approach (CSDfPCA) acts as a combined spatial-spectral filter (e.g., Tenke and Kayser, 2005), which identifies and separates latent variance unrelated to the spectral range of interest (e.g., artifacts related to blinks, eye movements, or muscle activity). Notably, the present low- and high-frequency alpha factors (i) did not include secondary loadings below $6 \mathrm{~Hz}$, and (ii) revealed topographies distinctly different from a blink topography (i.e., having posterior maxima in contrast to an anterior maximum).

Finally, the exclusively Caucausian, middle class and urban sample constitutes a limitation for the generalization of these findings. While we are not aware of prior EEG studies focusing on spectral differences as a function of ethnic, socioeconomic or cultural diversity, leave alone disparities in temporal stability, there is some evidence that theta and alpha oscillations during spontaneous self-referential thought may be affected by cross-cultural differences (Knyazev et al., 2012). Research in twins has emphasized the importance of genetic over environmental influences for EEG oscillations, particularly alpha (e.g., Begleiter and Porjesz, 2006; Ehlers et al., 2010; 
Lykken et al., 1982; Smit et al., 2006), and the association between alpha power and the gene for the corticotropin-releasing hormone binding protein is similar in Plains Indians and Caucasians (Enoch et al., 2008), suggesting that the reported findings are not likely due to the specific sample characteristics.

\section{Conclusions}

Overall posterior alpha, as quantified by reference-free CSD-fPCA, showed excellent temporal stability for resting EEG over a 9-16 yr time interval. These findings provide strong evidence that posterior alpha is a trait characteristic in adults, and can accordingly serve as a state-independent biomarker for MDD risk and prediction of treatment success and/or spontaneous recovery following pharmacological (e.g., SSRI) or other (e.g., placebo) interventions.

\section{Acknowledgments}

We dedicate this work to our colleague and friend, Dr. Craig E. Tenke, who passed away suddenly during the review process. Craig Tenke was instrumental for all aspects of this research and was the driving force behind the electrophysiological methods for quantifying EEG spectra. He will be greatly missed.

This work was supported by grants from the John Templeton Foundation (54679) and the National Institute of Mental Health (MH106905, MH036197). A summary of these data was presented at the 57th Annual Meeting of the Society of Psychophysiological Research (SPR), Vienna, Austria, October 11-15, 2017.

We would like to thank three anonymous reviewers for their helpful comments and critical feedback.

\section{Conflict of interest}

None.

\section{References}

Alhaj H. Wisniewski G, McAllister Williams RH. The use of the EEG in measuring therapeutic drug action: focus on depression and antidepressants. J Psychopharmacol 2011;25(9):1175-91.

Alschuler DM, Tenke CE, Bruder GE, Kayser J. Identifying electrode bridging from electrical distance distributions: a survey of publicly-available EEG data using a new method. Clin Neurophysiol 2014;125(3):484-90.

Barry RJ, De Blasio FM. EEG frequency PCA in EEG-ERP dynamics. Psychophysiology 2018;55(5):e13042. https://doi.org/10.1111/psyp.13042 [2017 Dec 11 Epub ahead of print].

Beauchaine TP. The role of biomarkers and endophenotypes in prevention and treatment of psychopathological disorders. Biomark Med 2009;3(1):1-3.

Begleiter H, Porjesz B. Genetics of human brain oscillations. Int J Psychophysiol 2006;60(2):162-71.

Bendat JS, Piersol AG. Random data: analysis and measurement procedures. New York, NY: Wiley-Interscience; 1971.

Benninger C, Matthis P, Scheffner D. EEG development of healthy boys and girls. Results of a longitudinal study. Electroencephalogr Clin Neurophysiol 1984;57 (1):1-12.

Berger H. Über das Elektroenkephalogramm des Menschen. Arch Psychiatr Nervenkr 1929;87:527-70.

BioSemi, Inc. ActiveTwo - multichannel, DC amplifier, 24-bit resolution, biopotential measurement system with active electrodes. Amsterdam, NL; 2001. <http://www.biosemi.com>.

Bruder GE, Fong R, Tenke CE, Leite P, Towey JP, Stewart JE, et al. Regional brain asymmetries in major depression with or without an anxiety disorder: a quantitative electroencephalographic study. Biol Psychiatry 1997;41 (9):939-48.

Bruder GE, Tenke CE, Warner V, Nomura Y, Grillon C, Hille J, et al. Electroencephalographic measures of regional hemispheric activity in offspring at risk for depressive disorders. Biol Psychiatry 2005;57(4):328-35.

Bruder GE, Tenke CE, Warner V, Weissman MM. Grandchildren at high and low risk for depression differ in EEG measures of regional brain asymmetry. Biol Psychiatry 2007;62(11):1317-23.

Bruder GE, Sedoruk JP, Stewart JW, McGrath PJ, Quitkin FM, Tenke CE. Electroencephalographic alpha measures predict therapeutic response to a selective serotonin reuptake inhibitor antidepressant: pre- and post-treatment findings. Biol Psychiatry 2008;63(12):1171-7.

Bruder GE, Bansal R, Tenke CE, Liu J, Hao X, Warner V, et al. Relationship of resting EEG with anatomical MRI measures in individuals at high and low risk for depression. Hum Brain Mapp 2012;33(6):1325-33.

Bruder GE, Tenke CE, Kayser J. Electrophysiological predictors of clinical response to antidepressants. In: Mann JJ, Rose SP, McGrath PJ, editors. The clinical handbook for the management of mood disorders. New York: Cambridge University Press; 2013. p. 380-93.

Doppelmayr M, Klimesch W, Pachinger T, Ripper B. Individual differences in brain dynamics: important implications for the calculation of event-related band power. Biol Cybern 1998;79(1):49-57.

Ehlers CL, Gizer IR, Phillips E, Wilhelmsen KC. EEG alpha phenotypes: linkage analyses and relation to alcohol dependence in an American Indian community study. BMC Med Genet 2010;11:43.

Enoch MA, Shen PH, Ducci F, Yuan Q, Liu J, White KV, et al. Common genetic origins for EEG, alcoholism and anxiety: the role of CRH-BP. PLOS ONE 2008;3 (10):3620.

Gottesman II, Gould TD. The endophenotype concept in psychiatry: etymology and strategic intentions. Am J Psychiatry 2003;160(4):636-45.

Gould TD, Gottesman II. Psychiatric endophenotypes and the development of valid animal models. Genes Brain Behav 2006;5(2):113-9.

Hao X, Talati A, Shankman SA, Liu J, Kayser J, Tenke CE, et al. Stability of cortical thinning in persons at increased familial risk for major depression across eight years. Biol Psychiatry Cogn Neurosci Neuroimaging 2017;2(7):619-25.

Jurcak V, Tsuzuki D, Dan I. 10/20,10/10, and 10/5 systems revisited: their validity as relative head-surface-based positioning systems. Neurolmage 2007;34 (4):1600-11.

Kayser J. Current source density (CSD) interpolation using spherical splines: CSD toolbox; 2009. Matlab code available at <http://psychophysiology. cpmc.columbia.edu/Software/CSDtoolbox>.

Kayser J, Tenke CE. Optimizing PCA methodology for ERP component identification and measurement: theoretical rationale and empirical evaluation. Clin Neurophysiol 2003;114(12):2307-25.

Kayser J, Tenke CE. Principal components analysis of Laplacian waveforms as a generic method for identifying ERP generator patterns: I. Evaluation with auditory oddball tasks. Clin Neurophysiol 2006a;117(2):348-68.

Kayser J, Tenke CE. Electrical distance as a reference-free measure for identifying artifacts in multichannel electroencephalogram (EEG) recordings. Psychophysiology 2006b;43:S51. <http://psychophysiology.cpmc.columbia. edu/mmedia/spr2006/ElecDistArti.pdf>.

Kayser J, Tenke CE. Hemifield-dependent N1 and event-related theta/delta oscillations: an unbiased comparison of surface Laplacian and common EEG reference choices. Int J Psychophysiol 2015a;97(3):258-70.

Kayser J, Tenke CE. Issues and considerations for using the scalp surface Laplacian in EEG/ERP research: a tutorial review. Int J Psychophysiol 2015b;97(3):189-209.

Knyazev GG, Savostyanov AN, Volf NV, Liou M, Bocharov AV. EEG correlates of spontaneous self-referential thoughts: a cross-cultural study. Int J Psychophysiol 2012;86(2):173-81.

Korb AS, Hunter AM, Cook IA, Leuchter AF. Rostral anterior cingulate cortex theta current density and response to antidepressants and placebo in major depression. Clin Neurophysiol 2009;120(7):1313-9.

Lykken DT, Tellegen A, Iacono WG. EEG spectra in twins: evidence for a neglected mechanism of genetic determination. Physiol Psychol 1982;10(1):60-5.

Miller L, Warner V, Wickramaratne P, Weissman M. Religiosity and depression: tenyear follow-up of depressed mothers and offspring. J Am Acad Child Adolesc Psychiatry 1997;36(10):1416-25.

Miller L, Bansal R, Wickramaratne P, Hao X, Tenke CE, Weissman MM, et al. Neuroanatomical correlates of religiosity and spirituality: a study in adults at high and low familial risk for depression. JAMA Psychiatry 2014;71(2):128-35.

Näpflin M, Wildi M, Sarnthein J. Test-retest reliability of resting EEG spectra validates a statistical signature of persons. Clin Neurophysiol 2007;118 (11):2519-24.

NeuroScan, Inc. SCAN 4.3 - vol. II. EDIT 4.3 - offline analysis of acquired data (Document number 2203, Revision D). Compumedics Neuroscan, El Paso, TX; 2003.

Perrin F, Pernier J, Bertrand O, Echallier JF. Spherical splines for scalp potential and current density mapping [Corrigenda EEG 02274, Electroenceph Clin Neurophysiol 1990;76:565]. Electroenceph Clin Neurophysiol 1989;72 (2):184-7.

Pivik RT, Broughton RJ, Coppola R, Davidson RJ, Fox N, Nuwer MR. Guidelines for the recording and quantitative analysis of electroencephalographic activity in research contexts. Psychophysiology 1993;30(6):547-58.

Pizzagalli D, Pascual Marqui RD, Nitschke JB, Oakes TR, Larson CL, Abercrombie HC, et al. Anterior cingulate activity as a predictor of degree of treatment response in major depression: evidence from brain electrical tomography analysis. Am J Psychiatry 2001;158(3):405-15.

Pizzagalli DA. Frontocingulate dysfunction in depression: toward biomarkers of treatment response. Neuropsychopharmacology 2011;36(1):183-206.

Salinsky MC, Oken BS, Morehead L. Test-retest reliability in EEG frequency analysis. Electroenceph Clin Neurophysiol 1991;79(5):382-92.

Smit DJ, Posthuma D, Boomsma DI, Geus EJ. Heritability of background EEG across the power spectrum. Psychophysiology 2005;42(6):691-7.

Smit CM, Wright MJ, Hansell NK, Geffen GM, Martin NG. Genetic variation of individual alpha frequency (IAF) and alpha power in a large adolescent twin sample. Int J Psychophysiol 2006;61(2):235-43. 
Tenke CE, Kayser J. A convenient method for detecting electrolyte bridges in multichannel electroencephalogram and event-related potential recordings. Clin Neurophysiol 2001;112(3):545-50.

Tenke CE, Kayser J. Reference-free quantification of EEG spectra: combining current source density (CSD) and frequency principal components analysis (fPCA). Clin Neurophysiol 2005;116(12):2826-46.

Tenke CE, Kayser J. Generator localization by current source density (CSD): implications of volume conduction and field closure at intracranial and scalp resolutions. Clin Neurophysiol 2012;123(12):2328-45.

Tenke CE, Kayser J. Surface Laplacians (SL) and phase properties of EEG rhythms: simulated generators in a volume-conduction model. Int J Psychophysiol 2015;97(3):285-98.

Tenke CE, Kayser J, Manna CG, Fekri S, Kroppmann CJ, Schaller JD, et al. Current source density measures of electroencephalographic alpha predict antidepressant treatment response. Biol Psychiatry 2011;70(4):388-94.

Tenke CE, Kayser J, Miller L, Warner V, Wickramaratne P, Weissman MM, et al. Neuronal generators of posterior EEG alpha reflect individual differences in prioritizing personal spirituality. Biol Psychol 2013;94(2):426-32.

Tenke CE, Kayser J, Abraham K, Alvarenga JE, Bruder GE. Posterior EEG alpha at rest and during task performance: comparison of current source density and field potential measures. Int J Psychophysiol 2015;97(3):299-309.

Tenke CE, Kayser J, Pechtel P, Webb CA, Dillon DG, Goer F, et al. Demonstrating testretest reliability of electrophysiological measures for healthy adults in a multisite study of biomarkers of antidepressant treatment response. Psychophysiology 2017a;54(1):34-50.
Tenke CE, Kayser J, Svob C, Miller L, Alvarenga JE, Abraham K, et al. Association of posterior EEG alpha with prioritization of religion or spirituality: a replication and extension at 20-year follow-up. Biol Psychol 2017b;124:79-86.

Tomarken AJ, Davidson RJ, Wheeler RE, Kinney L. Psychometric properties of resting anterior EEG asymmetry: temporal stability and internal consistency. Psychophysiology 1992;29(5):576-92.

Trivedi MH, McGrath PJ, Fava M, Parsey RV, Kurian BT, Phillips ML, et al. Establishing moderators and biosignatures of antidepressant response in clinical care (EMBARC): rationale and design. J Psychiatr Res 2016;78:11-23.

Ulrich G, Renfordt E, Frick K. The topographical distribution of alpha-activity in the resting EEG of endogenous-depressive inpatients with and without clinicalresponse to pharmacotherapy. Pharmacopsychiatry 1986;19:272-3.

Weissman MM, Warner V, Wickramaratne P, Moreau D, Olfson M. Offspring of depressed parents. 10 years later. Arch Gen Psychiatry 1997;54(10):932-40.

Weissman MM, Wickramaratne P, Nomura Y, Warner V, Verdeli H, Pilowsky DJ, et al. Families at high and low risk for depression: a 3-generation study. Arch Gen Psychiatry 2005;62(1):29-36.

Weissman MM, Berry OO, Warner V, Gameroff MJ, Skipper J, Talati A, et al. A 30-year study of 3 generations at high risk and low risk for depression. JAMA Psychiatry 2016a;73(9):970-7.

Weissman MM, Wickramaratne P, Gameroff MJ, Warner V, Pilowsky D, Kohad RG, et al. Offspring of depressed parents: 30 years later. Am J Psychiatry 2016b;173 (10):1024-32. 